\title{
Detecting Community Structures Within Complex Networks Using a Discrete Unconscious Search Algorithm
}

\author{
Ehsan Ardjmand, Ohio University, USA \\ William A. Young II, Ohio University, USA \\ Najat E. Almasarwah, Ohio University, USA
}

\begin{abstract}
Detecting the communities that exist within complex social networks has a wide range of application in business, engineering, and sociopolitical settings. As a result, many community detection methods are being developed by researchers in the academic community. If the communities within social networks can be more accurately detected, the behavior or characteristics of each community within the networks can be better understood, which implies that better decisions can be made. In this paper, a discrete version of an unconscious search algorithm was applied to three widely explored complex networks. After these networks were formulated as optimization problems, the unconscious search algorithm was applied, and the results were compared against the results found from a comprehensive review of state-of-the-art community detection methods. The comparative study shows that the unconscious search algorithm consistently produced the highest modularity that was discovered through the comprehensive review of the literature.
\end{abstract}

\section{KEYWORDS}

Evolutionary Searched-Based Algorithms, Modularity, Social Networks, Unweighted and Undirected Networks

\section{INTRODUCTION}

The amount of research dedicated to complex networks has increased over the last decades due to its wide range of applications. For example, by researching complex networks, the research community has investigated various phenomena within economic, social, and technological settings (Chen \& Redner, 2010). Within these problem areas, social structures can also be represented as a complex network. Social networks consist of a set of communities, which are sometimes referred to as called clusters or modules. This particular area of study is also receiving more attention from the research community. For example, Internet social networks (Lambiotte \& Ausloos, 2005), scientific reference networks (Chen \& Redner, 2010), biological networks (Diao, Li, Feng, Yin, \& Pan, 2007), neurological networks (Schwarz, Gozzi, \& Bifone, 2008), epidemiology networks (Sun \& Gao, 2007), transportation networks (Barigozzi, Fagiolo, \& Mangioni, 2011), and even political networks (Porter, Mucha, Newman, \& Friend, 2007) are some of the areas being investigated by practitioners and researchers.

Detecting the structure of the communities within a network receives a lot of attention from the research community because it plays a vital role in understanding the behavior among the various communities within a network (Gleich \& Seshadhri, 2012). To detect the structure of the communities within a complex network, a clustering operation is used (Zhou, Yang, Xie, Yang, \& Huang, 2019). 
As a result, decision-makers can make better, more informed, and more effective decisions depending on the application of the complex network being investigated.

Detecting communities in a social network is an NP-hard problem. Given this complexity, several methods have been proposed in the literature involving this particular problem. For example, spectral methods, divisive methods, agglomerative methods, maximization of the modularity, local methods, method selection, and roles of vertices are methods dedicated to detecting the structure of the communities within complex networks (Costa, Rodrigues, Travieso, \& Villas Boas, 2007). The unconscious search (US) algorithm is a method that is designed to tackle the continuous optimization problems (Ardjmand, Park, Weckman, \& Amin-Naseri, 2014). However, the unconscious search algorithm can be modified to solve discrete optimization problems. In this paper, the US algorithm is explored as a community detection method. Using the US algorithm as a method for detecting community structures has not been previously explored by researchers. Thus, the focus of the research presented in this paper involves a comprehensive comparative study of how well the US method preforms when comparing its results against other state-of-the-art methods found within recent academic research.

The comprehensive comparative study presented in this paper is primarily based on the concept of modularity, which is a popular measure in community detection literature. This measure is utilized to determine the strength of the division of a network. In other words, to detect the structure of the communities within a social network, a network $N$ can be partitioned in a manner that creates a subset of nodes containing a maximum number of internal links while minimizes the number of possible links with nodes outside of a given cluster (Girvan \& Newman, 2002).

\section{LITERATURE REVIEW}

Several methods have appeared in the literature that attempt to define the communities hidden within complex networks. These methods include, but are certainly not limited to the spectral bisection algorithm (Fiedler, 1973), Kernighan-Lin algorithm (Kernighan \& Lin, 1970), spectral partitioning (Elsner, 1997; Fjällström, 1998), and hierarchical clustering (Wasserman \& Faust, 1994). As noted, detecting community structures within complex networks is an emerging field of study. As a result, it is not surprising that the methods explored by researchers are quite diverse and the creativity exhibited from the research community is not lacking. For example, the theory of dynamics of excitable systems has used to developed a method to detect community structures (Li, Li, Hu, Di, \& Fan, 2010). Also, a novel cellular learning automate-based algorithm was developed to tackle the community detection problem within social networks that were both undirected and unweighted (Khomami, Rezvanian, \& Meybodi, 2018).

Detecting communities within social networks associate itself naturally to clustering methods. For example, a dynamic clustering method that utilizes a cluster's desynchronization properties of phase oscillators has been explored (Boccaletti, Ivanchenko, Latora, Pluchino, \& Rapisarda, 2007). It is quite common to find clustering methods being used to detect communities within structures. At times, the fundamental properties of the clusters are used to identify the communities within a network. However, clustering methods have also been used to enhance the development of community detection algorithms. For example, $k$-means clustering has been used in conjunction with a simulated annealing metaheuristic algorithm to detect communities within complex networks (Liu \& Liu, 2010). Clustering methods are classified as unsupervised learning methods. Although they are commonly used to detect communities within networks, they are not the only unsupervised learning methods that have been used by researchers. For example, a vector partitioning approach was adopted to detect community structures within network graphs (Wang, Shen, \& Ouyang, 2008).

In terms of community detection methods, many researchers have developed their methodologies around the premise of maximizing the modularity of a network, but they differ in their approach (Gach \& Hao, 2013). For example, a simulated annealing algorithm has been used as a method to 
maximize the modularity within a network (Medus, Acuna, \& Dorso, 2005). Though the concept of maximizing modularity has been integrated into many methods throughout the years, it still is a popular choice among researchers exploring more flexible algorithms to tackle the community detection problem (Zhou et al., 2019).

Research articles often focus on comparing the benefits and limitations of community detection methods. One consideration that researchers must consider is a method's ability to accurately detect the communities within networks. They must also take into account the time required to implement and produce accurate clusters within large networks. Some methods have been noted to be computationally expensive, while others were successfully applied to large datasets. For example, one method maximizing the modularity measure was applied to a network of 'collaboration among physicists' which was composed of about 50 thousand nodes (Newman, 2004). The researchers concluded that the algorithm was highly capable of detecting community structures in a short amount of time relative to other benchmark methods explored. Other methods can be found in the literature that aims to rapidly locate the core of each cluster residing inside a network (Chen, Fu, \& Shang, 2009). In addition, large undirected and unweighted networks with linear time complexity have also been used to examine an online community structure (Pan, Wangsheng, Zhaohui, \& Shijian, 2014).

From an efficiency standpoint, detecting communities within excessively large networks can be a considerable challenge that researchers must overcome. For example, label propagation has been used and was found to be an efficient approach from a computational time perspective when detecting the structure of the communities within excessively large networks (Liu \& Murata, 2010). However, traditional agglomerative algorithms have also been applied successfully to extremely large networks as well (Clauset, Newman, \& Moore, 2004). Although agglomerative approaches have been introduced as a method to determine community structures some time ago, they remain a popular choice among active researchers. For example, a degenerate agglomerative hierarchical clustering algorithm based on a reachability matrix was recently introduced by researchers (Maria Fiscarelli, Brust, Danoy, \& Bouvry, 2019).

Even though modularity remains to be the primary measure when determining the structure of communities within networks, it is certainly not the only measure being used by researchers. For example, a maximum flow factor has been utilized within community detection methods that are designed to eliminate the weakest links within a network (Mann, Matula, \& Olinick, 2008). Methods of this nature have the benefit of simplifying the overall structure of the complex network being investigated. A measure of node similarity has also been applied to complex networks and it was considered to be a fast and efficient method for detecting community structures within complex networks (Pan, Li, Liu, \& Liang, 2010). Other methodologies that use a measure other than modularity also exist. For example, a method leveraging structural similarity was applied to unweighted and undirected networks with promising results (Dabaghi Zarandi \& Kuchaki Rafsanjani, 2018). In addition, a communicability measure, which was inspired by how an epidemic pattern propagates through a network (Estrada \& Hatano, 2008, 2009; Rajtmajer \& Vukičević, 2010), has been used to determine a network's structure. It is clear from the literature, that there is not a shortage of inspiration when it comes to creating various measures detecting the communities within structures. For example, information discrepancy has also been used as a measure for community detection. Like other measures, it aims to determine the quality of detecting communities within complex networks (Zhang, Zhang, \& Zhang, 2008).

As noted, one consistent theme found in the literature is that many methods are developed to maximizing the modularity measure within complex networks. However, it is becoming more popular for researchers to develop community detection algorithms that utilize more than one measure. In addition to modularity, many examples within the literature can be found that formulate the structure of networks with other measures. For example, researchers have presented a parallel partitioning strategy based on two distinct measures for community detection. More specifically, researchers have developed an approach that accounts for the modularity of a network as well as a network's 
community recursive coefficient (Shen, Pei, Wang, Li, \& Wang, 2008). Another hybrid approach uses the affinity between nodes to determine the stability among the communities that are detected. This particular method strikes a balance between normalized mutual information and modularity to discover the communities hidden within complex networks (Zeng, Chenxi Shao, Xingfu Wang, \& Miao, 2020). Many other hybrid systems can also be found in the literature. For example, a community detection algorithm was developed that utilizes a graphlet measure to determine the structure of a social network (Abduljabbar, Hashim, \& Sallehuddin, 2020). For this novel work, the authors proposed two contradictory optimization functions, which are inspired by the cooperation between the neighborhood relationships and the signature similarity measures. In terms of another hybrid approach, attracting and recommending degree measures were utilized within an AR-clustering framework to strengthen the similarities among the vertices within social networks (Zhou, Li, Li, Zhang, \& Cui, 2017).

Although clustering methods are classified as unsupervised learning methods, the act of detecting communities within social networks is not limited to traditional clustering-based approaches. For example, a semi-supervised method for solving network community structure detection problems have also been introduced (Ma, Gao, Yong, \& Fu, 2010). The problems relating to community detection have also been formulated as optimization problems (Duch \& Arenas, 2005), which has led many researchers to formulate additional community detection methods based on evolutionary algorithms. Evolutionary algorithms are emerging in the literature for community detection and they often utilize measures of modularity to investigate the structure of complex networks (Guerrero, Montoya, Baños, Alcayde, \& Gil, 2017). One reason genetic algorithms are becoming a popular choice among researchers is because of their ability to model large complex networks (Li, Liu, \& Lao, 2013) and it is clear that these methods continue to evolve in their performance (Moradi \& Parsa, 2019). For example, quantum-inspired evolutionary algorithms are now being applied to very complex social networks (Yuanyuan \& Xiyu, 2018). Many other forms of evolutionary-based methods are now being explored. For example, a clonal section algorithm that maximizes modularity has been developed (Cai, Gong, Ma, \& Jiao, 2015). In addition, a discrete bat algorithm has been used to estimate the global optimality of community detection within complex networks (Song, Mingbo, Xuehai, Wei, $\& \mathrm{Ke}, 2016$ ). Finally, a novel multi-objective optimization algorithm using an ant colony algorithm was used to confront the community detection problem (Shahabi Sani, Manthouri, \& Farivar, 2018).

The research dedicated to social networks has increased dramatically over the last decade. Research relating to social networks is becoming more popular to cope with a great variety of phenomena and systems that have been classified as complex systems. Additionally, due to the sensitivity of the information on social networks, further privacy issues should be also considered. The main applications to the social network are the telecommunication industry, marketing purposes, online communities, search engines, etc. For these reasons and areas of application, methods dedicated to detecting community structures within complex networks are needed. Therefore, the motivation of the research contained in this article relates to exploring a new method for detecting community structures within complex networks that have not been previously explored. The dissemination of this research is considered significant given the novelty and the overall accuracy that was obtained by testing the performance of the algorithm on several complex networks that regularly appear in research dedicated to this specific topic. Investigating new methods of detecting community structures is significant because they can ultimately lead to advances in several areas of engineering, business, and other technology-focused fields of study. The evolutionary search-based method proposed in this article is based on the extremal optimization method (Duch \& Arenas, 2005). The method employs the discrete version of an optimization algorithm known as the unconscious search (US) algorithm (Ardjmand \& Amin-Naseri, 2012) to search for the optimum value of modularity.

The remainder of the article is organized as follows: Section 3 is devoted to a discussion of the US algorithm. Section 4 contains the results of the US method to popular benchmark networks that often appear in research exploring new methods for community detection. These commonly referenced networks include the 'Computer-Generated,' the 'Karate Club,' and the 'Dolphin' networks. Finally, 
Section 5 contains the conclusions reached by the authors using the US method as a community detection method with respect to the comprehensive comparative investigation that was performed.

\section{METHODOLOGY}

The methodology in this paper is based on the maximization of network modularity (Q) by using an unconscious search (US) algorithm. In the following sections, the concepts of community and modularity are introduced and the proposed algorithm is described.

\section{Community}

A community is represented by a group of nodes, which can be fully or partially interconnected to other nodes within the cluster (Newman, 2006). Figure 1 depicts a general representation of a structure with three distinct communities. In general, there are more connections that link the nodes of a community together than there are links connecting the communities within a network (Kenward \& Carrott, 2006). For example, the connections in groups A, B, and C are denser than between groups. There are 6,11 , and 10 between groups $\mathrm{A}, \mathrm{B}$, and $\mathrm{C}$, respectively.

Mathematically speaking, a network $N$ may be represented by a set of nodes $\left\{n_{i} \mid i=1, \ldots, n\right\}$ and a set of links $\left\{l_{i j} \mid i=1, \ldots, n, j=1, \ldots, n\right\}$. In this particular framework, $l_{i j}=0$ if there are no connections between the corresponding nodes $n_{i}$ and $n_{j}$. Thus, $l_{i j}=1$ if a connection is present. Complex networks are usually characterized by their edge weights. Considering these characteristics, complex networks can be classified into two types, unweighted networks and weighted networks (Buttner \& Krieter, 2018). In an unweighted network, the edge weights are ignored, and the structure of the graph defines the characteristics of the network. In a weighted network, the edge weights and structure of the graph play an important role in understanding the behavior of the network being investigated. Additionally, the networks can be classified as either undirected networks or directed networks. In an undirected network, there is only one edge that connects two notes together. This is unlike directed networks where there could be multiple edges between certain nodes (Newman, 2003b). Our proposed algorithm in this paper investigates unweighted and undirected networks, where $l_{i j}=l_{j i}$.

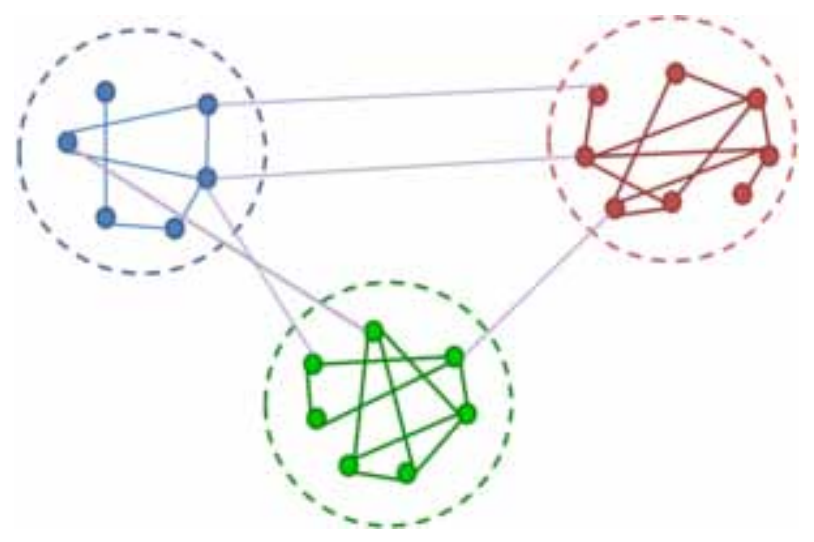




\section{Modularity (Q)}

For example, a typical partition $P$ of network $N$ is composed of $p$ communities $P_{1}, P_{2}, \ldots, P_{p}$. Considering a $p \times p$ symmetric matrix $e$ where, $e_{i j}$ denotes the ratio of all edges that connected vertices in $P_{i}$ to vertices in $P_{j}$. Furthermore, the trace of matrix $e\left(T r e=\sum_{i} e_{i i}\right)$ presents the fraction of edges in the network, and $\left|e^{2}\right|$ estimates the sum of all elements of the matrix $e$ Newman, 2003a; Newman \& Girvan, 2004). Thus, modularity can be calculated by Equation 1, which is shown below:

$$
Q=\sum_{i} e_{i i}-\sum_{i j k} e_{i j} e_{k i}=T r e-\left|e^{2}\right|
$$

In Equation 1, the modularity is represented by $Q$ and it is indicative of the ratio of internal links in a given community to the total number of links less than the expected value of the same ratio for the case where the links are distributed randomly across the network (Fan et al., 2018). Thus, the value of $Q$ must lie within the range between -1 and +1 . If the value is closer to 1 , it implies that $P$ can reflect the quality of modularity in a graph. As a result, the problem of detecting the community structure in a network comes down to choosing the partition $P$ such that the corresponding value of $Q$ is maximized. The degree of modularity can be also determined by Equation 2, were $L$ represents the total number of links in the network and $l_{s}$ signifies the number of links in the $\mathrm{s}^{\text {th }}$ module, while $d_{s}$ denotes the total of the degrees of nodes in the $\mathrm{s}^{\text {th }}$ module:

$$
Q=\sum_{s=1}^{p}\left[\frac{l_{s}}{L}-\left(\frac{d_{s}}{2 L}\right)^{2}\right]
$$

\section{The Unconscious Search Algorithm}

The unconscious search (US) algorithm is a meta-heuristic propounded by Ardjmand and AminNaseri (2012) and later adopted and applied by Xing and Gao (2014) and Molina et al. (2020). The US algorithm is a multi-start, memory-based metaheuristic that mimics the process of psychoanalytic psychotherapy proposed by Sigmund Freud, where the therapist tried to find the root cause of a patient's mental disorder based on their unconscious (Freud \& Strachey, 1996).

In each step, the US algorithm maintains a list of the best solutions that have been found in a set called a measurement matrix (MM). Once solutions are found, the US algorithm tries to find a good starting point and direction for the next search. For this purpose, US uses two types of measures, which are called displacement and condensational memories. Displacement memory attempts to learn the most promising starting point, while condensational memory attempts to find the most promising search directions. Whenever US finds a solution, it compares the performance results produced by the solutions within the MM against the performance of new solutions. In this manner, only the best performing solutions are stored, and the algorithm continues to test solutions until there is not an improvement to its modularity.

The US algorithm has four main steps, which include construction, construction review, a local search, and a measurement matrix update. In the construction step, a new solution is constructed. In the construction review, a solution is generated based on the results from the prior solutions that were found. These candidate solutions are based on a search that shows a promising direction. A local search 
enhances the best solution found in the first two phases, while in the last step, the measurement matrix is updated. The construction review phase in the US is mainly designed for continuous optimization problems. Several studies have utilized the US method to solve problems. For example, it has been used to solve an uncapacitated facility location problem (Ardjmand et al., 2014), and it has been used to solve a production planning with price uncertainty problem (Ardjmand, Weckman, Young, Sanei Bajgiran, \& Aminipour, 2016). However, the proposed methodology presented in this article is applied to discrete problems (i.e. number of clusters), which means that this particular phase is not needed (Ardjmand et al., 2014). As a result, the proposed US algorithm will only have three phases, which include construction, a local search, and a measurement matrix update.

To apply the US to community detection problems within complex networks, a network is initially divided into two partitions in such a way that the modularity measure is maximized. Next, the two partitions are divided into two sub-partitions to ensure that the modularity of each sub-partition is maximized. The procedure is repeated, using the US algorithm, until the modularity measure, which is shown in Equation 2, is not improved upon. The process of using the US algorithm for the community detection problem is summarized in Figure 2.

In the first iteration of the US algorithm, a set of random solutions (i.e. a network with nodes randomly assigned to two clusters) is generated. These solutions are stored in a measurement matrix (MM). The size of the MM is shown by $|M M|$ and this measurement defines the number of bestperforming solutions that are stored. Thus, if $|M M|=10$, the 10 best-performing solutions are stored.

To construct a new solution, the US assigns each node of the network to a cluster. For example, based on the best solutions stored in MM, if a node is assigned to Cluster $160 \%$ of the time, and it is assigned to Cluster $240 \%$ of the time, then based on the new solution, the node will have a $60 \%$ chance of being assigned to Cluster 1 , and a $40 \%$ chance being assigned Cluster 2 . In terms of creating new solutions, this type of memory is referred to as displacement memory (Ardjmand \& Amin-Naseri, 2012).

Figure 2. US algorithm for Clustering Networks

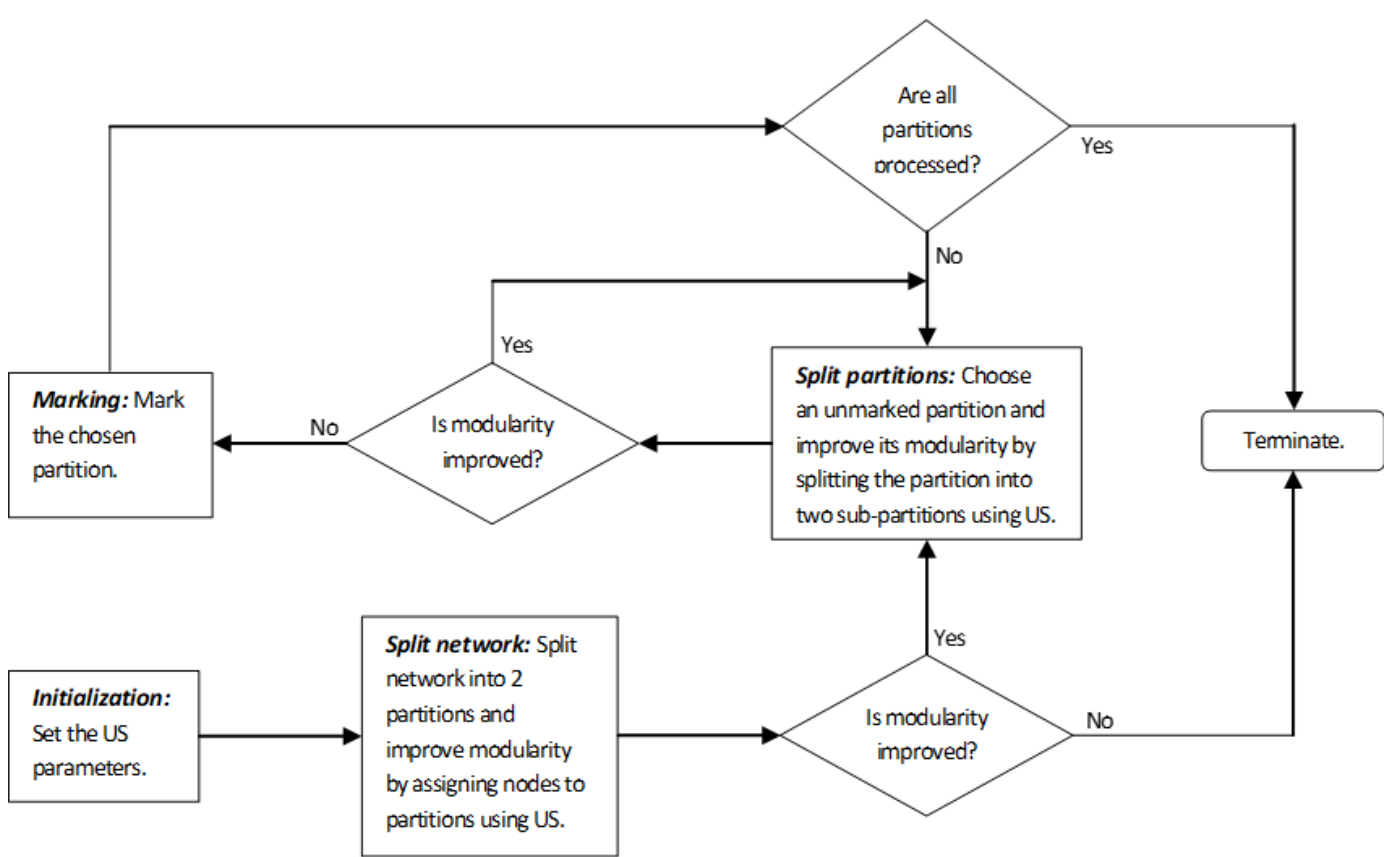


For the local search, after a solution is constructed, a node will be picked randomly and transferred from one cluster to another. If this move improves the modularity, the assignment will be accepted. Otherwise, if this is not the case, the solution will be rejected. This process continues until the modularity ceases to improve any further.

The last step of the US algorithm is the local search. In this step, if a given solution is an improvement over the worst-performing solution stored in MM, the new solution will be inserted in $\mathrm{MM}$ and the worst performing solution found in the MM will be discarded. Likewise, if the new solution does not perform better than the worst solution stored in MM, it will be discarded, and the MM will not be updated.

\section{RESULTS}

To evaluate the performance of the US algorithm as a new community detection method, three complex networks were explored. These particular networks are commonly used by researchers exploring new methods for community detection. Given the popularity of these benchmark networks, the results obtained from the US algorithm were compared to the results published by authors exploring community detection methods. Computer-Generated Networks (CGN).

The first complex network that was investigated in this research was the Computer-Generated Network (CGN). CGN involves a series of graphs representing pre-identified community structures. The CGN consists of 128 nodes and four pre-defined clusters. Each node was linked with an average of $z_{\text {in }}$ number of nodes to the same cluster, and an average of $z_{\text {out }}$ number of nodes to other clusters, such that $z_{\text {in }}+z_{\text {out }}=16$. To produce measures that can be compared against other published results, a large number of graphs were created from the US algorithm that consisted of $z_{\text {out }}$ values that ranged between 0 to 10 . The CGN problem has been explored by many researchers. Figure 3 shows a summary of the results obtained by other researchers who investigated the CGN problem with various community detection algorithms. In particular, the graph shows the percentage of nodes that were correctly clustered by each community detection algorithm. The US algorithm's ability to detect community structures for values of $z_{\text {out }} \leq 8$ preformed just as well or better than the other results found in the literature. However, for $z_{\text {out }}>8$ the US outperformed all other methods recorded from the literature.

\section{Karate Club Network}

The Karate Club Network (KCN) problem was the second complex network that was explored. This network was first introduced by Zachary (1977) but has remained popular with researchers exploring community detection algorithms. The KCN consists of 34 nodes and 78 links, where the network represents the membership of a karate club and the friendship between the members. The objective of this problem is to detect the communities within the social network. When the US algorithm was applied to the KCN, it produced a modularity of 0.420 . The graph network based on the US solution is shown in Figure 4, where four communities or clusters were detected.

As noted, the $\mathrm{KCN}$ is popular among researchers investigating new community detection methods. To assess how well the US algorithm performed as a community detection method, a comprehensive review of the literature was conducted. As a result of this investigation, a total of 26 references were found that solved the $\mathrm{KCN}$ with a variety of community detection methods. The summary of this review is shown in Figure 5, where research utilizing the KCN dates back as far back as 2005 and includes studies as recent as 2020. The US algorithm was able to produce a modularity of 0.42 for the KCN network. In terms of this metric, the US algorithm was able to outperform many other techniques that were found during the comprehensive review of the literature. The US algorithm produced the highest modularity that has discovered during the review of existing literature exploring the KCN. However, as shown in Figure 4, many other methods were capable of producing this value of modularity as well. It should be noted that additional information about the studies shown in Figure 4 is provided in Section 5. 
Figure 3. Benchmark Results for the Computer-Generated Network

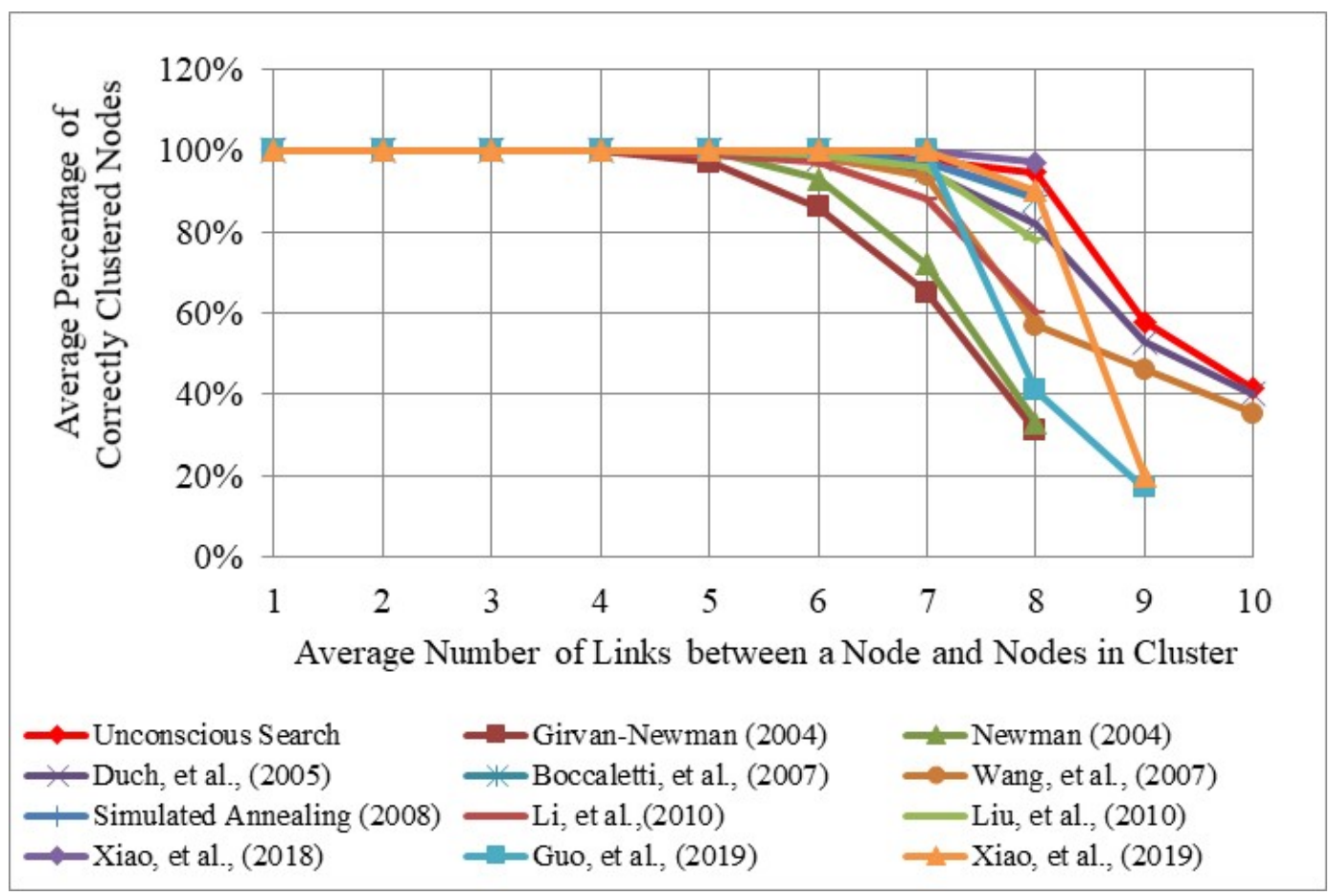

\section{Dolphin Network}

The third and final complex network that was evaluated in this study was the Dolphin Network. Just like the two previous networks investigated, this network has been utilized by many other researchers exploring community detection algorithms. The Dolphin Network problem involves a network of 62 nodes and 159 links, which represent the interaction between dolphins in the network. The US

Figure 4. Unconscious Search Algorithm Results for the Karate Club Network

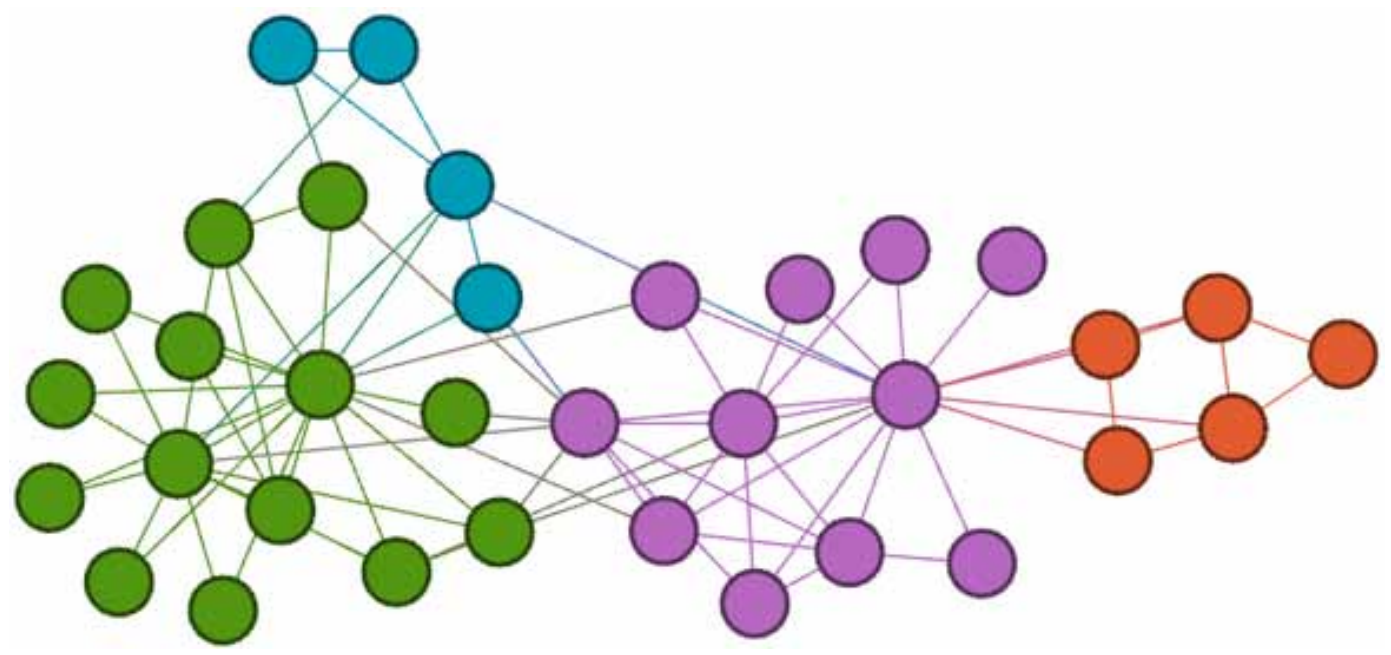




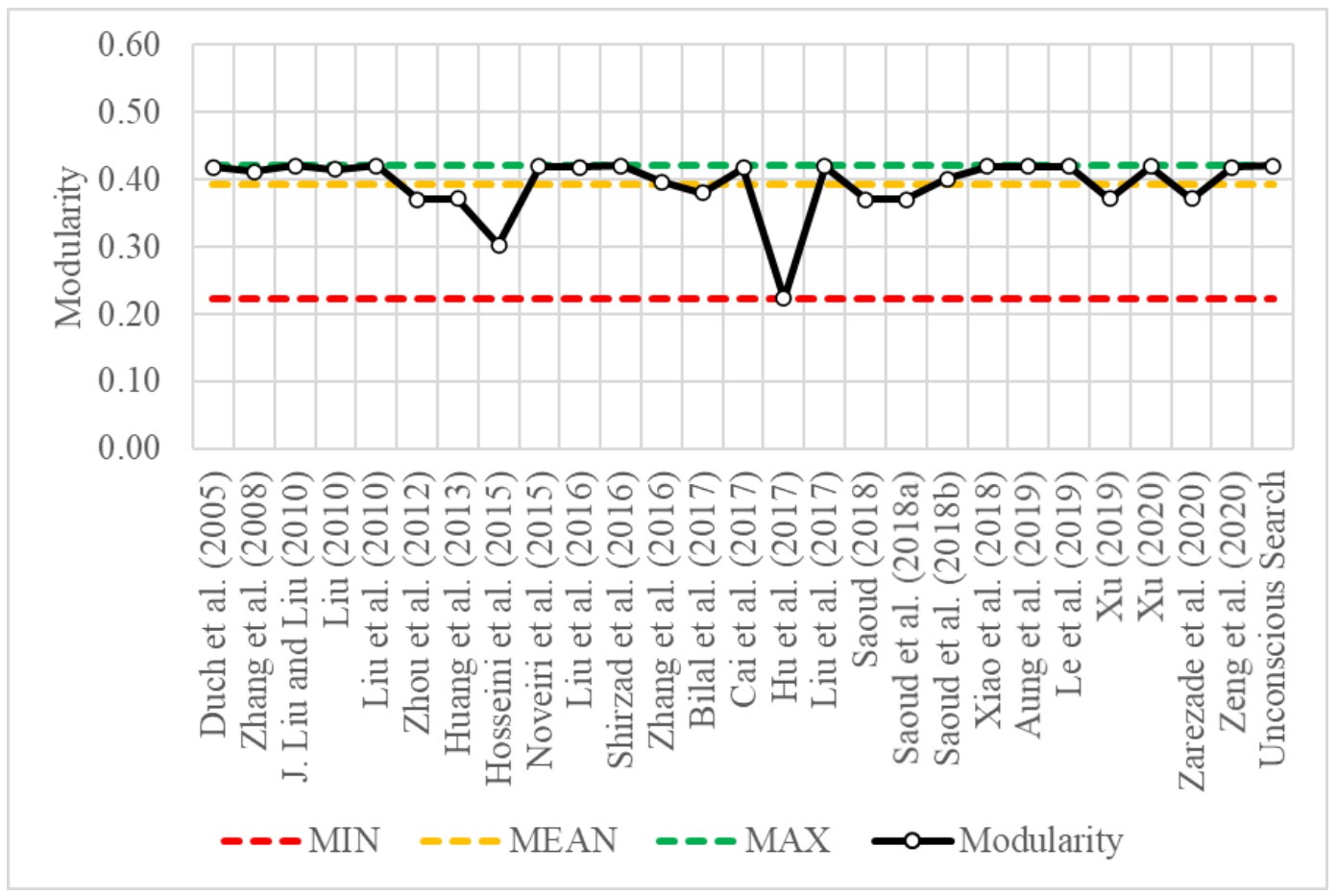

algorithm was used to partition the network into four subsets. The communities detected by the US algorithm are illustrated in Figure 6, where the resulting modularity was found to be 0.527 .

Given its complexity and size, the Dolphin Network is another network that is widely used by researchers exploring advances within community detection algorithms. In total, 24 references utilizing the Dolphin Network were found through the process of reviewing the literature. Based on the review of literature, the studies that were found that utilized the Dolphin Network ranged in years from 2010 to 2020. As shown in Figure 7, the US algorithm produced a modularity of 0.527 for the Dolphin Network. Based on the comparative study, these results were similar to the outcome of the KCN study. In other words, the US algorithm was able to outperform many other community detection methods; however, even though it produced the highest value of modularity that was observed, it was not the only method to do so.

\section{CONCLUSION}

The US algorithm is a multi-start, memory-based metaheuristic that mimics the process of psychoanalytic psychotherapy. In this article, the US algorithm was adapted to solve community detection problems of small dimensional scale. Several complex networks appear in the related literature surrounding community detection. Of these networks, three were selected and used to compare the results of the US algorithm to the results based on other state-of-the-art methodologies that have appeared recently in the literature. These networks included the Computer-Generated Network (CGN), the Karate Club Network (KCN), and the Dolphin Network.

With respect to the networks explored in this study, the use of the US algorithm showed promising results. For example, the US method was able to produce results that were among the highest, or in certain situations, was the highest performing method when applied as a community detection 


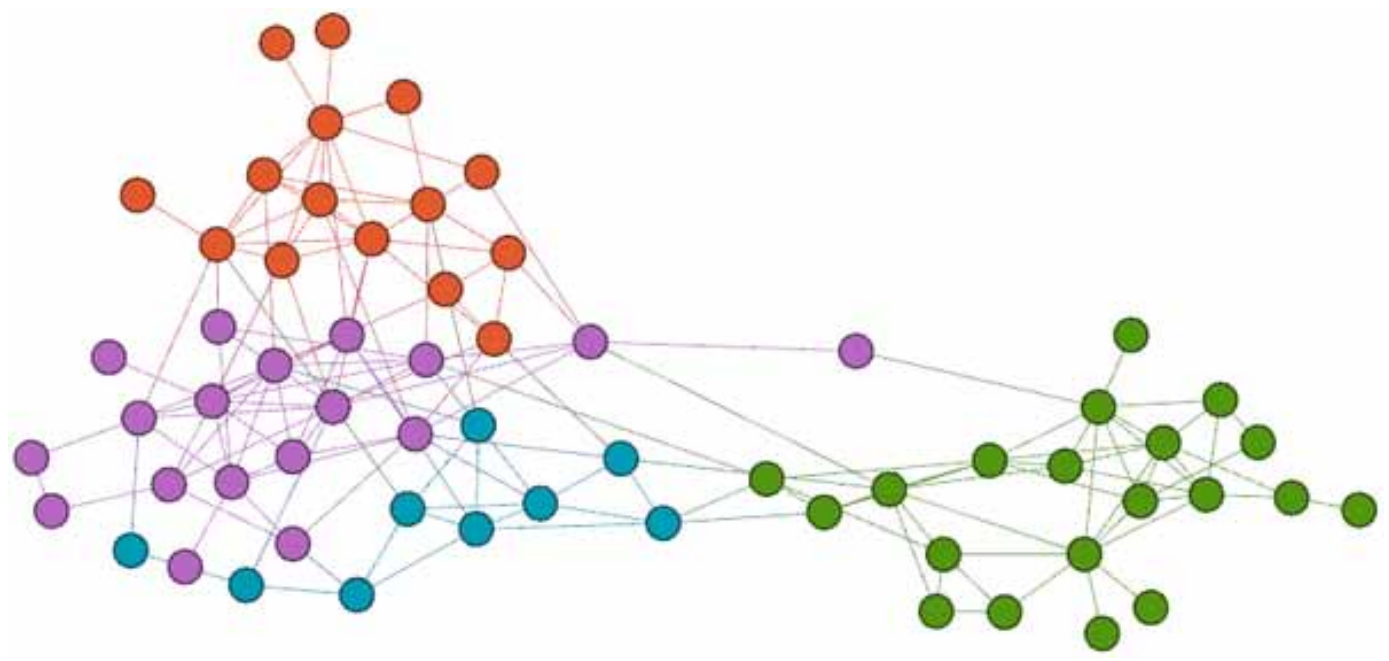

Figure 7. Benchmark Results for the Dolphin Network

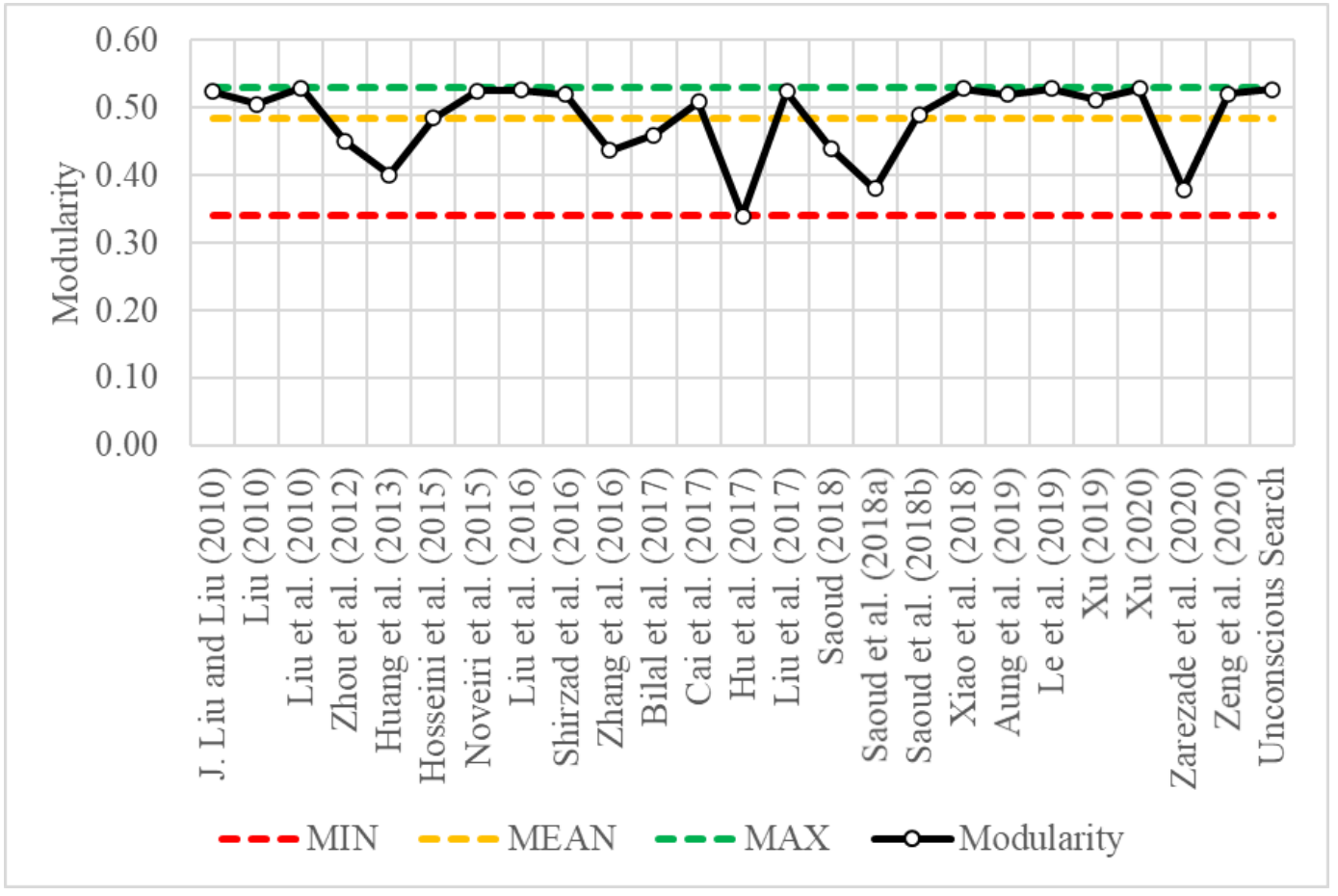

method to the CGN. For the KCN and Dolphin Network problems, the modularity obtained by the US algorithm performed equally as well as the best results that were discovered from a comprehensive review of related literature. Table 1 contains additional information related to the comprehensive review of state-of-the-art community detection methods for the KCN and the Dolphin Network. As the table shows, the US algorithm consistently produced highly accurate results. 
Table 1. Comparison Summary of Community Detection Algorithm

\begin{tabular}{|c|c|c|c|}
\hline Reference & $\begin{array}{c}\text { Karate } \\
\text { Club }\end{array}$ & Dolphin & Community Detection Method Description \\
\hline Zhou, et al., (2012) & 0.370 & 0.450 & Greedy Algorithm \\
\hline Saoud, et al., (2018b) & 0.401 & 0.490 & Detection Based on Node Similarity and Modularity \\
\hline Bilal, et al., (2017) & 0.380 & 0.460 & Evolutionary algorithm \\
\hline Huang, et al., (2013) & 0.371 & 0.401 & Multi-Agent based decentralized algorithm \\
\hline $\mathrm{Xu},(2019)$ & 0.372 & 0.511 & Communicability Distance Algorithm \\
\hline $\mathrm{Xu},(2020)$ & 0.419 & 0.528 & Spectral algorithm \\
\hline Aung, et al., (2019) & 0.419 & 0.520 & Discrete artificial bee colony algorithm \\
\hline Le, et al., (2019) & 0.419 & 0.528 & Meta-Heuristic based label propagation \\
\hline Zarezade, et al., (2020) & 0.371 & 0.379 & SD-GCN Algorithm \\
\hline Zeng, et al., (2020) & 0.418 & 0.521 & Affinity Between Nodes \& Stability of Community \\
\hline Xiao, et al., (2018) & 0.419 & 0.528 & Differential Evolution Algorithm \\
\hline Saoud, et al., (2018a) & 0.370 & 0.380 & Genetic Algorithm \\
\hline Liu, et al., (2017) & 0.419 & 0.524 & A Biogeography-based Optimization Algorithm \\
\hline $\mathrm{Hu}$, et al., (2017) & 0.223 & 0.340 & Novel Algorithm J-SC \\
\hline Cai, et al., (2017) & 0.417 & 0.508 & Particle Swarm Optimization Algorithm \\
\hline Liu, et al., (2016) & 0.418 & 0.526 & Genetic algorithm \\
\hline Zhang, et al., (2016) & 0.396 & 0.436 & Fuzzy community detection approach \\
\hline Hosseini, et al., (2015) & 0.303 & 0.485 & Memory-based Label Propagation Algorithm \\
\hline Noveiri, et al., (2015) & 0.419 & 0.524 & Ant Colony Algorithm and Fuzzy Clustering \\
\hline Liu, (2010) & 0.415 & 0.505 & Simulated Annealing Strategy \\
\hline Saoud, (2018) & 0.370 & 0.440 & Artificial Bee Colony \\
\hline Liu and Liu (2010) & 0.420 & 0.523 & Simulated Annealing with k-means Algorithms \\
\hline Duch, et al., (2005) & 0.418 & - & Extremal Optimization Method \\
\hline Liu, et al., (2010) & 0.420 & 0.529 & Label Propagation Algorithm \\
\hline Zhang, et al., (2008) & 0.411 & - & Iterative Procedure based on Information Discrepancy \\
\hline Shirzad, et al., (2016) & 0.420 & 0.520 & Hierarchical approach \\
\hline Unconscious Search & 0.420 & 0.527 & Unconscious Search Algorithm \\
\hline
\end{tabular}

In conclusion, the US algorithm was able to produce results that were consistently among the best results found from a comprehensive review of recent literature. The networks that were explored were small in scale; however, these networks are consistently used by the research community to judge how well algorithms discover the communities that are hidden within complex networks.

The downside of the algorithm was the local optimal solution. In this case, the immigration operator should be applied when there is no improvement in the generations due to the premature convergence to a local optimum. This relaxation helped the US algorithm to reach the optimal solution in many situations. Additionally, the research conducted in this article was restricted to small-scale unweighted and undirected networks. The proposed algorithm solved the three complex networks that were selected for this study in only a few minutes. In all cases that were explored, the US algorithm detected the communities within two minutes. Given the scope of the research conducted, 
the performance of the US algorithm on large-scale, weighted, and directed networks is unknown. Researchers are advised to take this under consideration until these types of are explored, Thus, future research will focus on using the US method to detect community structures within larger complex networks that are weighted and directed. 


\section{REFERENCES}

Abduljabbar, D. A., Hashim, S. Z. M., \& Sallehuddin, R. (2020). An improved multi-objective evolutionary algorithm for detecting communities in complex networks with graphlet measure. Computer Networks, 169, 107070. Advance online publication. doi:10.1016/j.comnet.2019.107070

Ardjmand, E., \& Amin-Naseri, M. R. (2012). Unconscious search-a new structured search algorithm for solving continuous engineering optimization problems based on the theory of psychoanalysis. Paper presented at the International Conference in Swarm Intelligence. doi:10.1007/978-3-642-30976-2_28

Ardjmand, E., Park, N., Weckman, G., \& Amin-Naseri, M. R. (2014). The discrete unconscious search and its application to uncapacitated facility location problem. Computers \& Industrial Engineering, 73, 32-40. doi:10.1016/j.cie.2014.04.010

Ardjmand, E., Weckman, G. R., Young, W. A. II, Sanei Bajgiran, O., \& Aminipour, B. (2016). A robust optimisation model for production planning and pricing under demand uncertainty. International Journal of Production Research, 54(13), 3885-3905. doi:10.1080/00207543.2016.1161251

Aung, T. T., \& Nyunt, T. T. S. (2019). Discrete Artificial Bee Colony Algorithm for Community Detection in Social Network. Paper presented at the Seventeenth International Conference on Computer Applications (ICCA 2019).

Barigozzi, M., Fagiolo, G., \& Mangioni, G. (2011). Identifying the community structure of the internationaltrade multi-network. Physica A, 390(11), 2051-2066. doi:10.1016/j.physa.2011.02.004

Bilal, S., \& Abdelouahab, M. (2017). Evolutionary algorithm and modularity for detecting communities in networks. Physica A, 473, 89-96. doi:10.1016/j.physa.2017.01.018

Boccaletti, S., Ivanchenko, M., Latora, V., Pluchino, A., \& Rapisarda, A. (2007). Detecting complex network modularity by dynamical clustering. Physical Review. E, 75(4), 045102. doi:10.1103/PhysRevE.75.045102 PMID:17500946

Buttner, K., \& Krieter, J. (2018). Comparison of weighted and unweighted network analysis in the case of a pig trade network in Northern Germany. Preventive Veterinary Medicine, 156, 49-57. doi:10.1016/j. prevetmed.2018.05.008 PMID:29891145

Cai, Q., Gong, M., Ma, L., \& Jiao, L. (2015). A novel clonal selection algorithm for community detection in complex networks. Computational Intelligence, 31(3), 442-464. doi:10.1111/coin.12031

Cai, X., Shi, Y., Zhu, Y., Qiao, Y., \& Hu, F. (2017). An Algorithm Q-PSO for Community Detection in Complex Networks. Paper presented at the 2017 16th International Symposium on Distributed Computing and Applications to Business, Engineering and Science (DCABES). doi:10.1109/DCABES.2017.23

Chen, D., Fu, Y., \& Shang, M. (2009). A fast and efficient heuristic algorithm for detecting community structures in complex networks. Physica A, 388(13), 2741-2749. doi:10.1016/j.physa.2009.03.022

Chen, P., \& Redner, S. (2010). Community structure of the physical review citation network. Journal of Informetrics, 4(3), 278-290. doi:10.1016/j.joi.2010.01.001

Clauset, A., Newman, M. E. J., \& Moore, C. (2004). Finding community structure in very large networks. Physical Review. E, 70(6), 066111. doi:10.1103/PhysRevE.70.066111 PMID:15697438

Costa, L. D. F., Rodrigues, F. A., Travieso, G., \& Villas Boas, P. R. (2007). Characterization of Complex Networks: A Survey of measurements. Advances in Physics, 56(1), 167-242. doi:10.1080/00018730601170527

Dabaghi Zarandi, F., \& Kuchaki Rafsanjani, M. (2018). Community detection in complex networks using structural similarity. Physica A, 503, 882-891. doi:10.1016/j.physa.2018.02.212

Diao, Y., Li, M., Feng, Z., Yin, J., \& Pan, Y. (2007). The community structure of human cellular signaling network. Journal of Theoretical Biology, 247(4), 608-615. doi:10.1016/j.jtbi.2007.04.007 PMID:17540409

Duch, J., \& Arenas, A. (2005). Community detection in complex networks using extremal optimization. Physical Review. E, 72(2), 027104. doi:10.1103/PhysRevE.72.027104 PMID:16196754

Elsner, U. (1997). Graph partitioning. Academic Press. 
Estrada, E., \& Hatano, N. (2008). Communicability in complex networks. Physical Review. E, 77(3), 036111.

Estrada, E., \& Hatano, N. (2009). Communicability graph and community structures in complex networks. Applied Mathematics and Computation, 214(2), 500-511. doi:10.1016/j.amc.2009.04.024

Fan, X., Chen, Z., Cai, F., Wu, J., Liu, S., Liao, Z., \& Liao, Z. (2018). Local Core Members Aided Community Structure Detection. Mobile Networks and Applications, 24(4), 1373-1381. doi:10.1007/s11036-018-0994-2

Fiedler, M. (1973). Algebraic connectivity of graphs. Czechoslovak Mathematical Journal, 23(2), 298-305. doi:10.21136/CMJ.1973.101168

Fjällström, P. O. (1998). Algorithms for graph partitioning. Linköping University Electronic Press.

Freud, S., \& Strachey, J. (1996). The interpretation of dreams. Gramercy Books.

Gach, O., \& Hao, J.-K. (2013). Improving the Louvain algorithm for community detection with modularity maximization. Paper presented at the International Conference on Artificial Evolution, Cham.

Girvan, M., \& Newman, M. E. (2002). Community structure in social and biological networks Paper presented at the national academy of sciences. doi:10.1073/pnas.122653799

Gleich, D. F., \& Seshadhri, C. (2012). Vertex neighborhoods, low conductance cuts, and good seeds for local community methods. Paper presented at the 8th ACM SIGKDD international conference on Knowledge discovery and data mining. doi:10.1145/2339530.2339628

Guerrero, M., Montoya, F. G., Baños, R., Alcayde, A., \& Gil, C. (2017). Adaptive community detection in complex networks using genetic algorithms. Neurocomputing, 266, 101-113. doi:10.1016/j.neucom.2017.05.029

Hosseini, R., \& Azmi, R. (2015). Memory-based label propagation algorithm for community detection in social networks. Paper presented at The International Symposium on Artificial Intelligence and Signal Processing (AISP). doi:10.1109/AISP.2015.7123488

Hu, F., Wang, M., Wang, Y., Hong, Z., \& Zhu, Y. (2017). An algorithm J-SC of detecting communities in complex networks. Physics Letters. [Part A], 381(42), 3604-3612. doi:10.1016/j.physleta.2017.09.013

Huang, J., Yang, B., Jin, D., \& Yang, Y. (2013). Decentralized mining social network communities with agents. Mathematical and Computer Modelling, 57(11-12), 2998-3008. doi:10.1016/j.mcm.2013.03.005

Kenward, H., \& Carrott, J. (2006). Insect species associations characterise past occupation sites. Journal of Archaeological Science, 33(10), 1452-1473. doi:10.1016/j.jas.2005.06.018

Kernighan, B. W., \& Lin, S. (1970). An Efficient Heuristic Procedure for Partitioning Graphs. The Bell System Technical Journal, 49(2), 291-307. doi:10.1002/j.1538-7305.1970.tb01770.x

Khomami, M. M. D., Rezvanian, A., \& Meybodi, M. R. (2018). A new cellular learning automata-based algorithm for community detection in complex social networks. Journal of Computational Science, 24, 413-426. doi:10.1016/j.jocs.2017.10.009

Lambiotte, R., \& Ausloos, M. (2005). Uncovering collective listening habits and music genres in bipartite networks. Physical Review. E, 72(6), 066107. doi:10.1103/PhysRevE.72.066107 PMID:16486010

Le, B.-D., Shen, H., Nguyen, H., \& Falkner, N. (2019). Improved network community detection using metaheuristic based label propagation. Applied Intelligence, 49(4), 1451-1466. doi:10.1007/s10489-018-1321-0

Li, X., Li, M., Hu, Y., Di, Z., \& Fan, Y. (2010). Detecting community structure from coherent oscillation of excitable systems. Physica A, 389(1), 164-170. doi:10.1016/j.physa.2009.09.006

Li, Y., Liu, G., \& Lao, S.-y. (2013). A genetic algorithm for community detection in complex networks. Journal of Central South University, 20(5), 1269-1276. doi:10.1007/s11771-013-1611-y

Liu, H., Yang, F., \& Liu, D. (2016). Genetic algorithm optimizing modularity for community detection in complex networks. Paper presented at the 35th Chinese Control Conference (CCC), Chengdu. doi:10.1109/ ChiCC.2016.7553259

Liu, J. (2010). Fuzzy modularity and fuzzy community structure in networks. The European Physical Journal $B, 77(4), 547-557$. doi:10.1140/epjb/e2010-00290-3 
Liu, J., \& Liu, T. (2010). Detecting community structure in complex networks using simulated annealing with k-means algorithms. Physica A, 389(11), 2300-2309. doi:10.1016/j.physa.2010.01.042

Liu, S., \& Li, Z. (2017). A Biogeography-Based Optimization Algorithm For Community Detection In Complex Networks Paper presented at the 2017 International Conference on Algorithms, Methodology, Models and Applications in Emerging Technologies (ICAMMAET). doi:10.1109/ICAMMAET.2017.8186746

Liu, X., \& Murata, T. (2010). Advanced modularity-specialized label propagation algorithm for detecting communities in networks. Physica A, 389(7), 1493-1500. doi:10.1016/j.physa.2009.12.019

Ma, X., Gao, L., Yong, X., \& Fu, L. (2010). Semi-supervised clustering algorithm for community structure detection in complex networks. Physica A, 389(1), 187-197. doi:10.1016/j.physa.2009.09.018

Mann, C. F., Matula, D. W., \& Olinick, E. V. (2008). The use of sparsest cuts to reveal the hierarchical community structure of social networks. Social Networks, 30(3), 223-234. doi:10.1016/j.socnet.2008.03.004

Maria Fiscarelli, A., Brust, M. R., Danoy, G., \& Bouvry, P. (2019). A vertex-similarity clustering algorithm for community detection. Journal of Information and Telecommunication, 4(1), 36-50. doi:10.1080/2475183 9.2019.1686683

Medus, A., Acuna, G., \& Dorso, C. (2005). Detection of community structures in networks via global optimization. Physica A, 358(2), 593-604. doi:10.1016/j.physa.2005.04.022

Molina, D., Poyatos, J., Del Ser, J., García, S., Hussain, A., \& Herrera, F. (2020). Comprehensive Taxonomies of Nature- and Bio-inspired Optimization: Inspiration versus Algorithmic Behavior, Critical Analysis and Recommendations. arXiv preprint arXiv:2002.08136

Moradi, M., \& Parsa, S. (2019). An evolutionary method for community detection using a novel local search strategy. Physica A, 523, 457-475. doi:10.1016/j.physa.2019.01.133

Newman, M. E. (2003a). Mixing patterns in networks. Physical Review E: Statistical, Nonlinear, and Soft Matter Physics, 67(2 Pt 2), 026126. doi:10.1103/PhysRevE.67.026126 PMID:12636767

Newman, M. E. (2003b). The structure and function of complex networks. SIAM Review, 45(2), 167-256. doi: $10.1137 / \mathrm{S} 003614450342480$

Newman, M. E. (2004). Fast algorithm for detecting community structure in networks. Physical Review E: Statistical, Nonlinear, and Soft Matter Physics, 69(6 Pt 2), 066133. doi:10.1103/PhysRevE.69.066133 PMID:15244693

Newman, M. E. (2006). Modularity and community structure in networks. Paper presented at the national academy of sciences. doi:10.1073/pnas.0601602103

Newman, M. E., \& Girvan, M. (2004). Finding and evaluating community structure in networks. Physical Review. E, 69(2), 15. doi:10.1103/PhysRevE.69.026113 PMID:14995526

Noveiri, E., Naderan, M., \& Alavi, S. E. (2015). Community detection in social networks using ant colony algorithm and fuzzy clustering. Paper presented at the 2015 5th International Conference on Computer and Knowledge Engineering (ICCKE). doi:10.1109/ICCKE.2015.7365864

Pan, G., Wangsheng, Z., Zhaohui, W., \& Shijian, L. (2014). Online Community Detection for Large Complex Networks. PLoS One, 9(7), e102799. Advance online publication. doi:10.1371/journal.pone.0102799 PMID:25061683

Pan, Y., Li, D.-H., Liu, J.-G., \& Liang, J.-Z. (2010). Detecting community structure in complex networks via node similarity. Physica A, 389(14), 2849-2857. doi:10.1016/j.physa.2010.03.006

Porter, M. A., Mucha, P. J., Newman, M. E. J., \& Friend, A. (2007). Community structure in the united states house of representatives. Physica A, 386(1), 414-438. doi:10.1016/j.physa.2007.07.039

Rajtmajer, S. M., \& Vukičević, D. (2010). A note on the Estrada-Hatano communicability algorithm for detecting community structure in complex networks. Applied Mathematics and Computation, 217(7), 3516-3521. doi:10.1016/j.amc.2010.09.026 
Saoud, B. (2018). Networks clustering with bee colony. Artificial Intelligence Review, 52(2), 1297-1309. doi:10.1007/s10462-018-9657-8

Saoud, B., \& Moussaoui, A. (2018a). A new hierarchical method to find community structure in networks. Physica A, 495, 418-426. doi:10.1016/j.physa.2017.12.095

Saoud, B., \& Moussaoui, A. (2018b). Node similarity and modularity for finding communities in networks. Physica A, 492, 1958-1966. doi:10.1016/j.physa.2017.11.110

Schwarz, A. J., Gozzi, A., \& Bifone, A. (2008). Community structure and modularity in networks of correlated brain activity. Magnetic Resonance Imaging, 26(7), 914-920. doi:10.1016/j.mri.2008.01.048 PMID:18479871

Shahabi Sani, N., Manthouri, M., \& Farivar, F. (2018). A multi-objective ant colony optimization algorithm for community detection in complex networks. Journal of Ambient Intelligence and Humanized Computing, 11(1), 5-21. doi:10.1007/s12652-018-1159-7

Shen, Y., Pei, W., Wang, K., Li, T., \& Wang, S. (2008). Recursive filtration method for detecting community structure in networks. Physica A, 387(26), 6663-6670. doi:10.1016/j.physa.2008.08.029

Shirzad, M., \& Feizi-Derakhshi, M.-R. (2016). Hierarchical community detection in social networks using spectral method. International Journal of Computer Science and Information Security, 14(8), 9.

Song, A., Mingbo, L., Xuehai, D., Wei, C., \& Ke, P. (2016). Community Detection Using Discrete Bat Algorithm IAENG. International Journal of Computational Science, 43(2), 7.

Sun, H., \& Gao, Z. (2007). Dynamical behaviors of epidemics on scale-free networks with community structure. Physica A, 381, 491-496. doi:10.1016/j.physa.2007.03.030

Wang, G., Shen, Y., \& Ouyang, M. (2008). A vector partitioning approach to detecting community structure in complex networks. Computers \& Mathematics with Applications (Oxford, England), 55(12), 2746-2752. doi:10.1016/j.camwa.2007.10.028

Wasserman, S., \& Faust, K. (1994). Social network analysis: Methods and applications (Vol. 8). Cambridge university press. doi:10.1017/CBO9780511815478

Xiao, J., Zhang, Y.-J., \& Xu, X.-K. (2018). Convergence improvement of differential evolution for community detection in complex networks. Physica A, 503, 762-779. doi:10.1016/j.physa.2018.02.072

Xing, B., \& Gao, W.-J. (2014). Innovative computational intelligence: a rough guide to 134 clever algorithms (Vol. 62). Springer. doi:10.1007/978-3-319-03404-1

Xu, Y. (2019). Community detection based on network communicability distance. Physica A, 515, 112-118. doi:10.1016/j.physa.2018.09.191

$\mathrm{Xu}, \mathrm{Y}$. (2020). A spectral method to detect community structure based on the communicability modularity. Physica A, 537, 122751. Advance online publication. doi:10.1016/j.physa.2019.122751

Yuanyuan, M., \& Xiyu, L. (2018). Quantum inspired evolutionary algorithm for community detection in complex networks. Physics Letters. [Part A], 382(34), 2305-2312. doi:10.1016/j.physleta.2018.05.044

Zachary, W. W. (1977). An information flow model for conflict and fission in small groups. Journal of Anthropological Research, 33(4), 452-473. doi:10.1086/jar.33.4.3629752

Zarezadeh, M., Nourani, E., \& Bouyer, A. (2020). Community Detection using a New Node Scoring and Synchronous Label Updating of Boundary Nodes in Social Networks. Journal of Artificial Intelligence and Data Mining.

Zeng, J., Shao, C., Wang, X., \& Miao, F. (2020). A new method for detecting communities in network basedon the affinity between nodes. Paper presented at the IOP.

Zhang, H., Chen, X., Li, J., \& Zhou, B. (2016). Fuzzy community detection via modularity guided membershipdegree propagation. Pattern Recognition Letters, 70, 66-72. doi:10.1016/j.patrec.2015.11.008

Zhang, J., Zhang, S., \& Zhang, X. S. (2008). Detecting community structure in complex networks based on a measure of information discrepancy. Physica A, 387(7), 1675-1682. doi:10.1016/j.physa.2007.10.061 
Zhou, H., Li, J., Li, J., Zhang, F., \& Cui, Y. (2017). A graph clustering method for community detection in complex networks. Physica A, 469, 551-562. doi:10.1016/j.physa.2016.11.015

Zhou, X., Yang, K., Xie, Y., Yang, C., \& Huang, T. (2019). A novel modularity-based discrete state transition algorithm for community detection in networks. Neurocomputing, 334, 89-99. doi:10.1016/j.neucom.2019.01.009

Zhou, Z., Wang, W., \& Wang, L. (2012). Community Detection Based on an Improved Modularity. Paper presented at the Chinese Conference on Pattern Recognition, Berlin, Germany. doi:10.1007/978-3-642-33506-8_78

Ehsan Ardjmand is an Assistant Professor of Analytics and Information Systems at Ohio University. His teaching and research interests include predictive and prescriptive modeling in business, machine learning, soft computing, decision support systems, and operations research. He earned his Ph.D. in Industrial and Systems Engineering from Ohio University. Dr. Ardjmand has more than eight years of experience in manufacturing and service industries in various operations management and analytics related positions. He has led several research projects related to business intelligence and data science and their applications in manufacturing, supply chain, social media, and stock markets, to name a few.

William A. Young II is a Charles G. O'Bleness Associate Professor of Business Analytics in the Department of Analytics and Information Systems at Ohio University. Young earned his doctorate degree in Mechanical and Systems Engineering from Ohio University in 2010. William also received a bachelor's and master's degree in Electrical Engineering at Ohio University in 2002 and 2005, respectively. William has collaborated with multidisciplinary teams of faculty, students, and professionals on projects and programs. Young's primary research and teaching interests relate to operation management, healthcare services, and environmental systems, as well as specific interests in quantitative sports analysis, and educational technologies and techniques for innovative curriculum development and teaching instruction.

Najat Almasarwah obtained her master's degree in industrial and systems engineering from Ohio University, and her BS degree in industrial and systems engineering from Mutah University, Jordan. Currently, she is a Ph.D. candidate of industrial and system engineering at Ohio University. Her research focuses on the cellular manufacturing systems design and control, pallet loading problem, industry 4.0, sustainability and data analysis. Almasarwah has published several papers in journals and conference proceedings and made conference presentations. 